\title{
Commissioning of the ATLAS detector and combined test beam results
}

P. Perrodo, LAPP-I N2P3-CNRS/ CERN, on behalf of the ATLAS collaboration

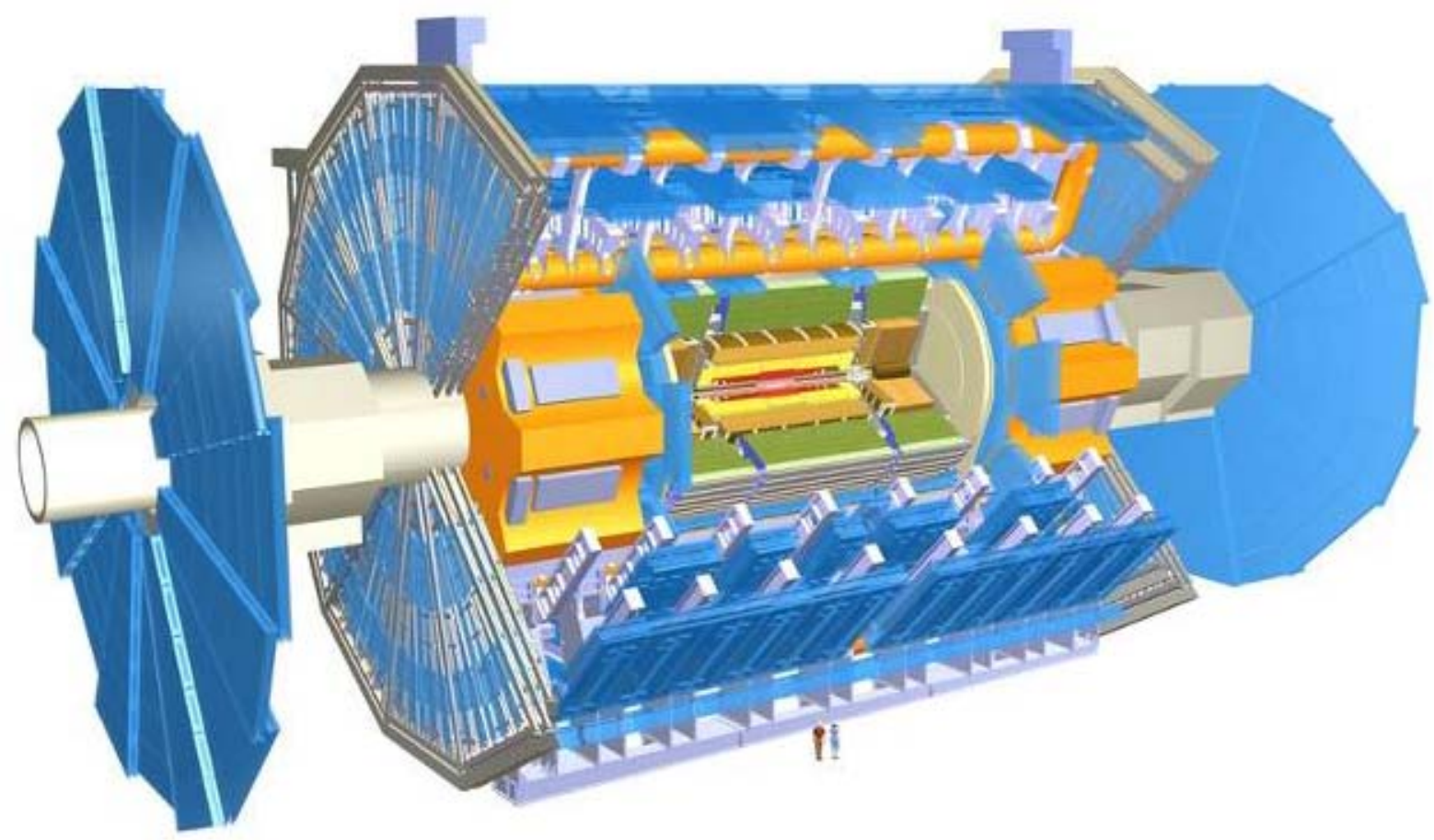

Xth Pisa Meeting, May 21-27 2006, I sola d'Elba 


\section{ATLAS road map}

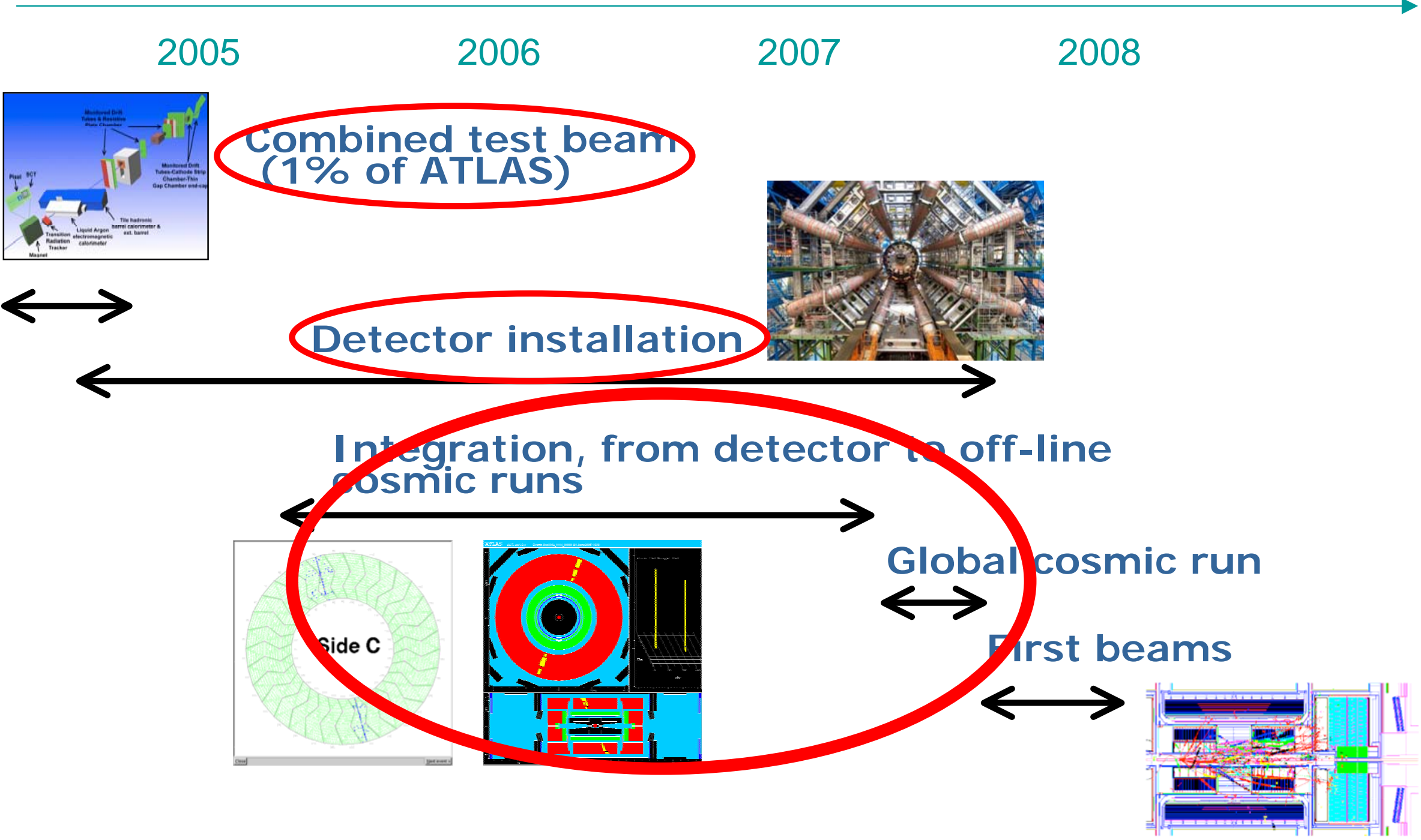


- Liquid argon and TILES calorimeters:

- All in the cavern, Barrel LARG cryostat is cold,

- Barrel Front end electronics all here, expect the power supplies

- A small fraction of the readout is possible yet

- Will grow in size with more power supplies

- Magnets:

- Muon spectrometer (barrel and forward)

- Chambers under installation.

- Solenoid: cold

- Very small fraction of the readout available

- Forward wheels coming next

- Inner detector (SCT+TRT, pixel later)

- SCT+TRT barrel integrated, tested on surface

- Endcap toroid: end Sept. 06

- Good fraction of the readout present already used.

- Then long installation in the cavern 


\section{ATLAS combined test beam}

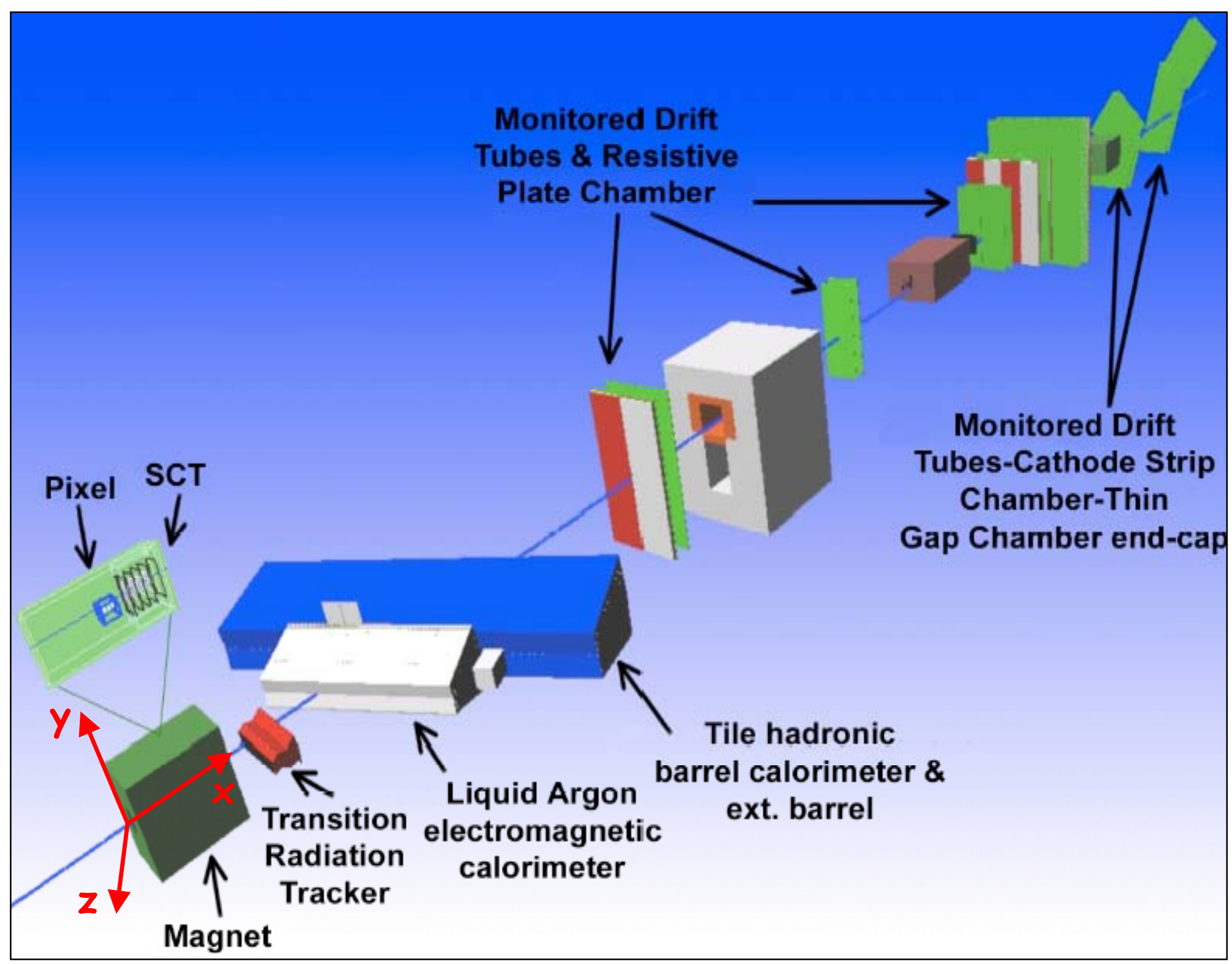




\section{Test beam results}

- Tracking Muon Spec.- I nner Detector

- Back extrapolation of a muon track to the inner detector ( Pixel + SCT)

- Validation of the reconstruction software

- Alignment procedure

- Measure of the tracking performances

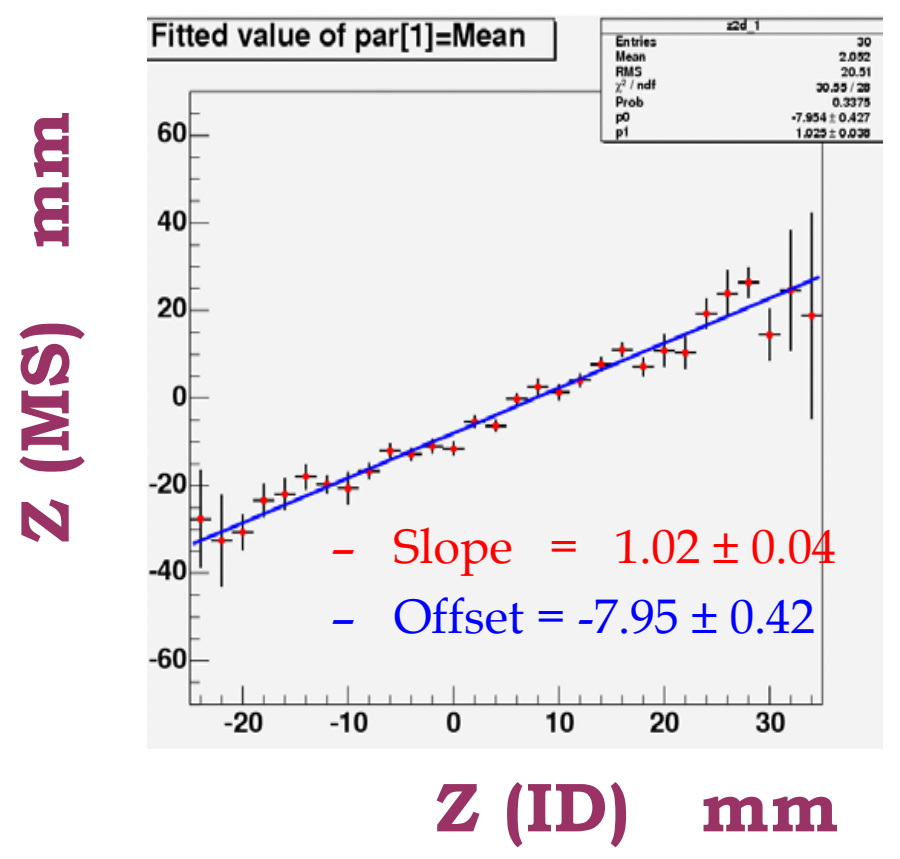

- Muons ( $300 \mathrm{GeV})$ with a Bremsstrahlung in the Calorimeters

- Validation of the reconstruction software

- Evaluate inter-calibration Calo Muon spectrometer

- Quality of the simulation (Geant 4)

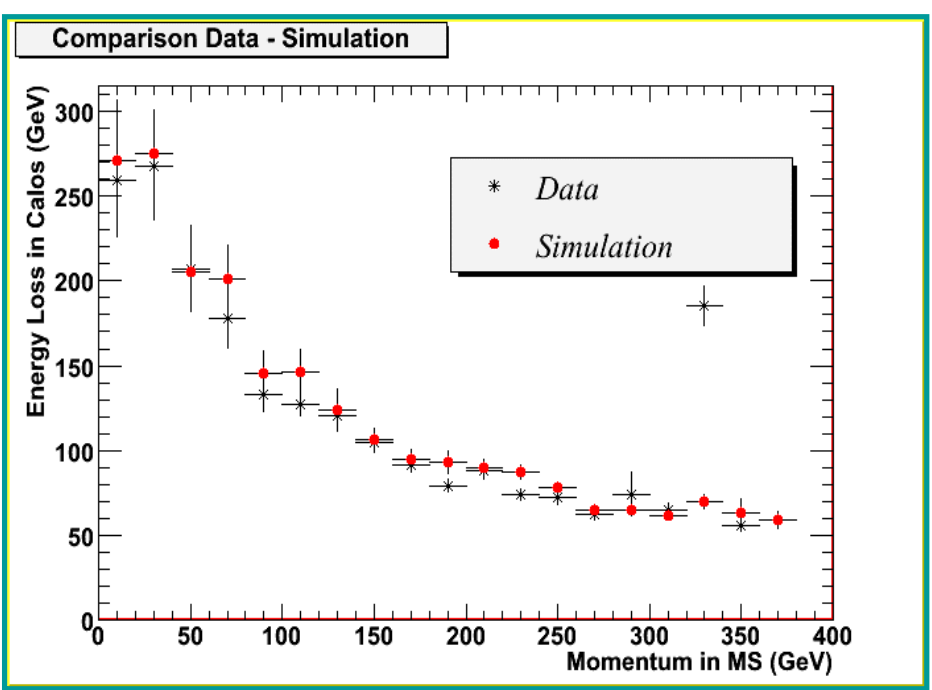


- Exercise various combinations of ATLAS sub-systems:

- Detectors, DAQ and online databases. Idem with DCS (Slow ctrl.)

- Calorimeters and the calorimeter trigger. Calibration, integration of the trigger.

- Magnets:

- Barrel toroid tests. Exercise all installed detectors. Functional tests. Cosmic runs.

- Solenoid mapping. Exercise the calorimeters electronics.

- Functional tests: test the performances: operational, new errors, recovery procedures, stability of the data taking, calibration procedures

\section{- Run with cosmics:}

- Data taking, on line monitoring, full analysis chain exercised. Detector study: bad channels.

- Repeat the exercise when the readout system grows in size. 


\section{Detector schematics}

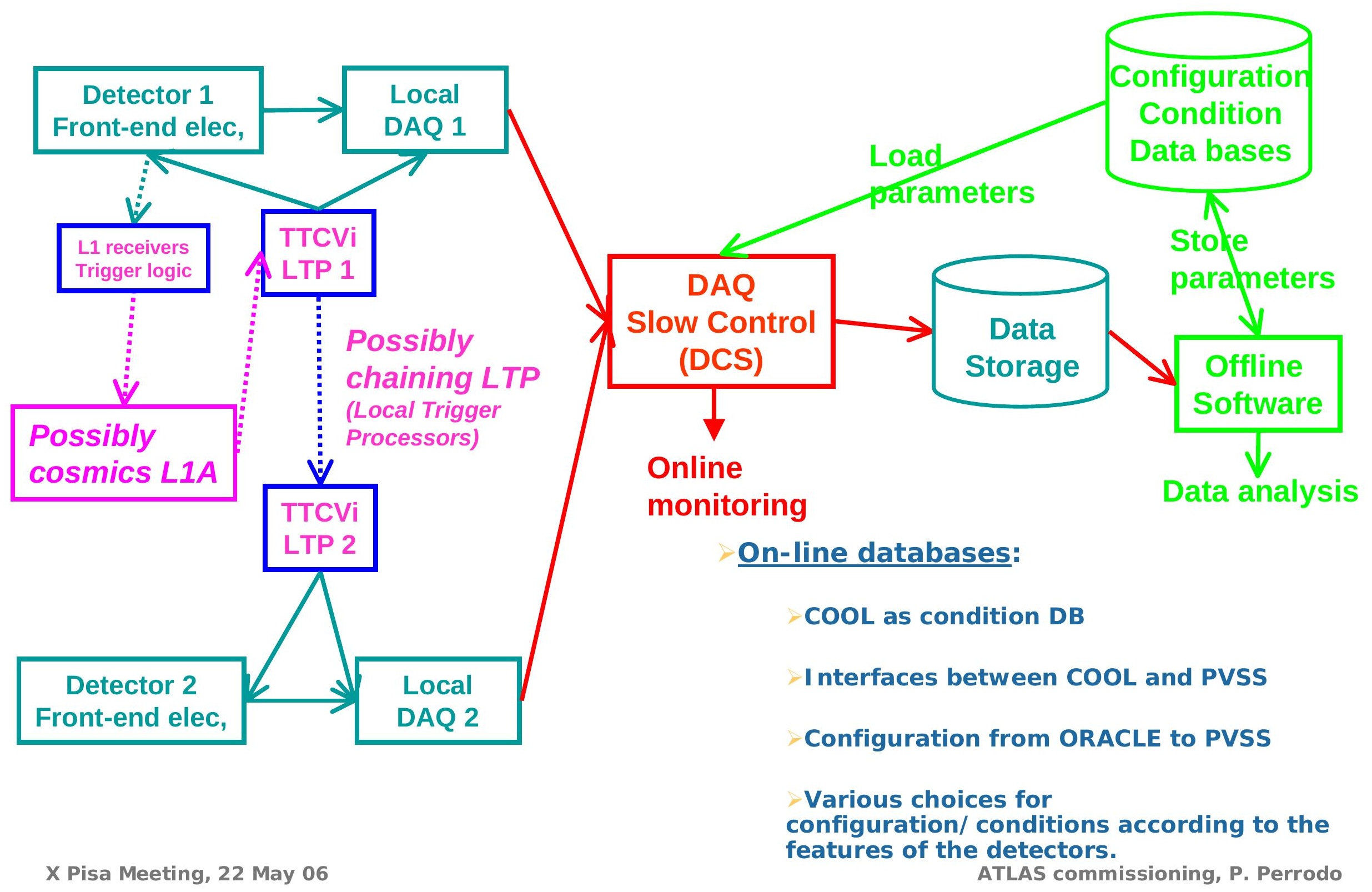




\section{DAQ components}

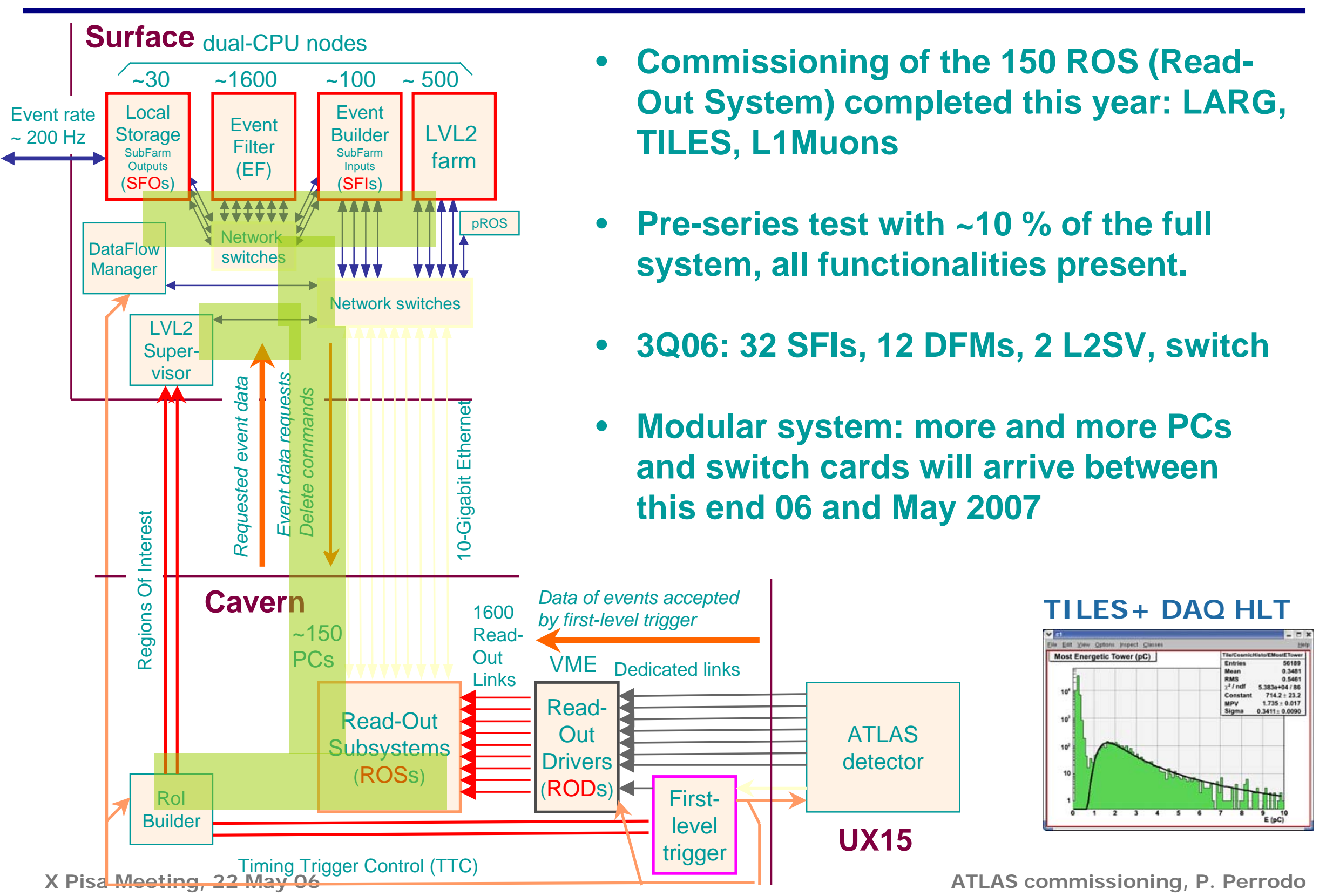




\section{DAQ pre series results}

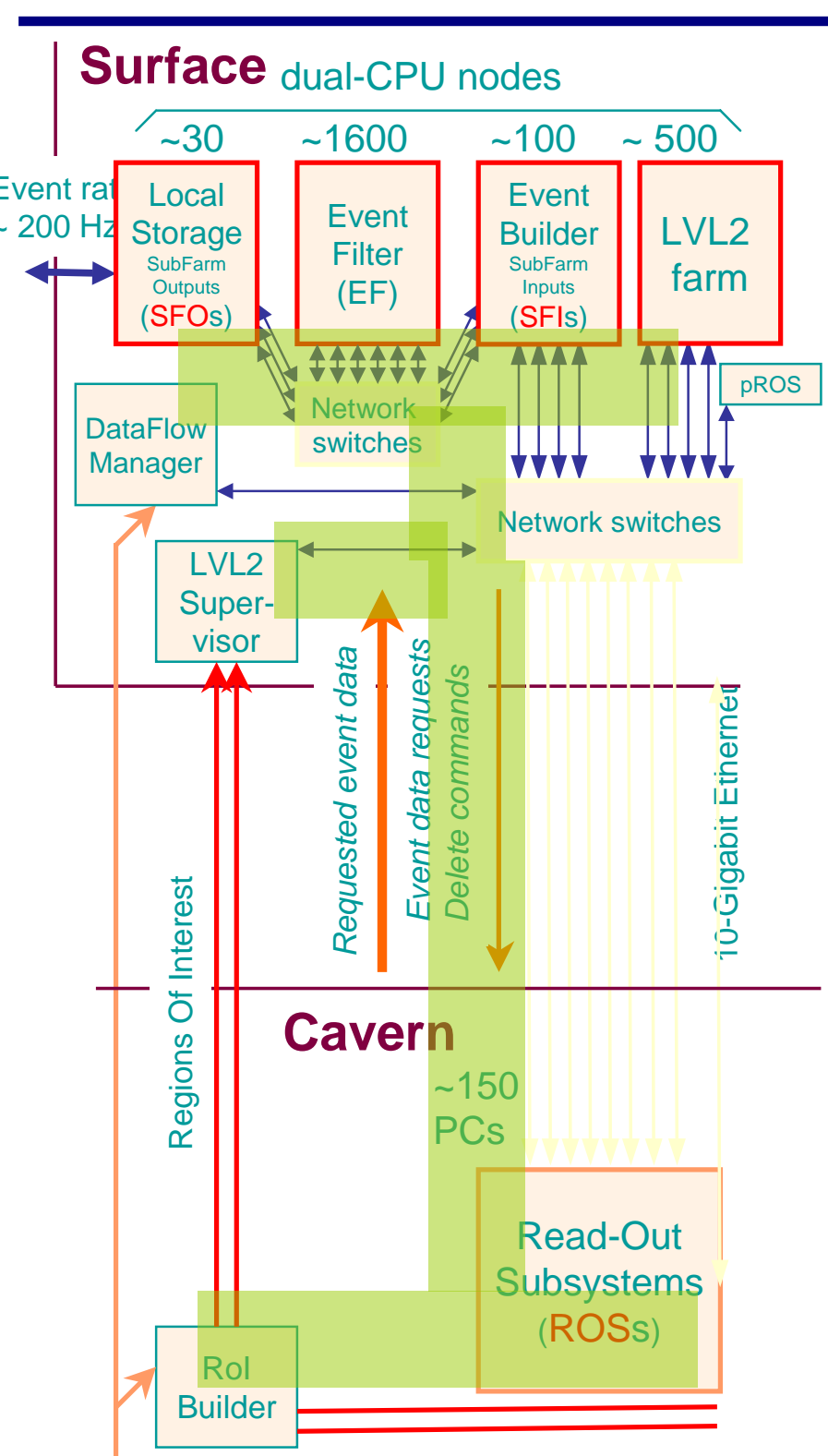

X Pisa Manting Timing Trigger Control (TTC)
- Event Builder only. Comparison pre-series with model in various configurations.

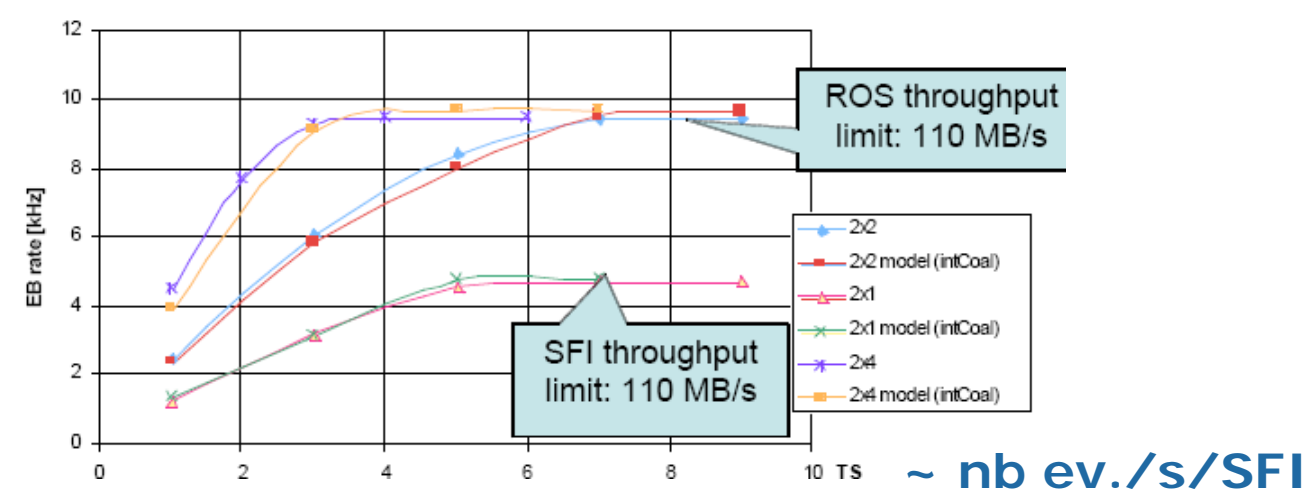

- Event Builder + Level2. Dummy L2 algorithm. 8 ROS, 8 SFI. Comparison pre-series with model in various configurations.

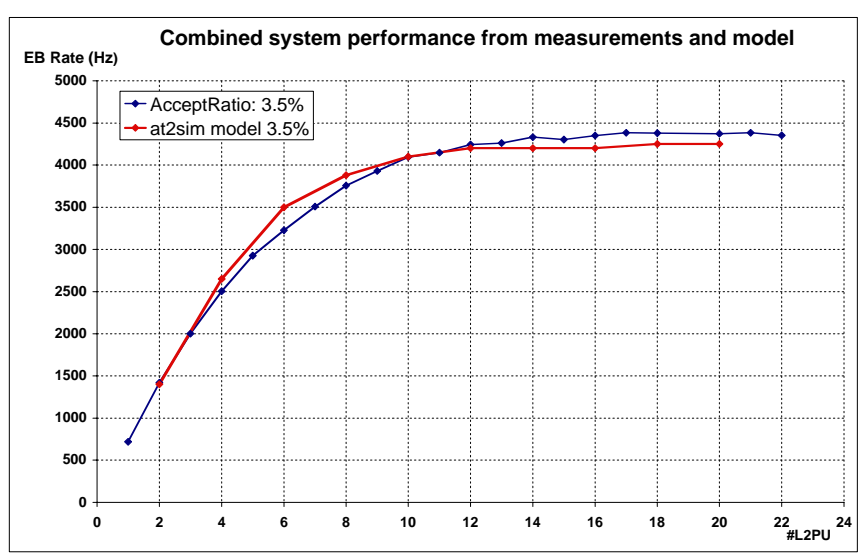

ATLAS commissioning, P. Perrodo 


\section{DAQ pre series results}

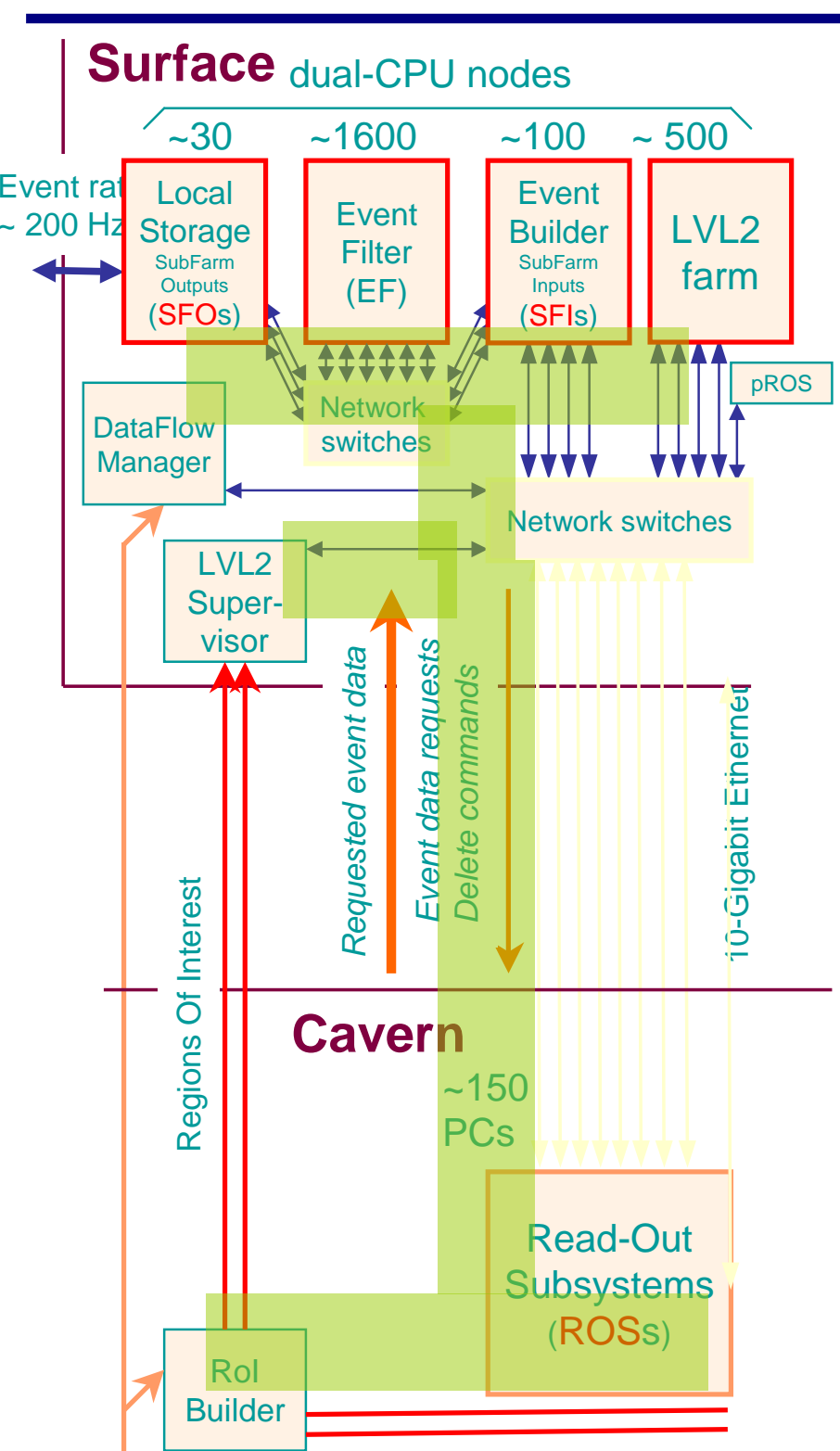

- Long runs (->24hrs). 8 ROS, 8 SFI, 20 L2, dummy algorithms. Stability observed.

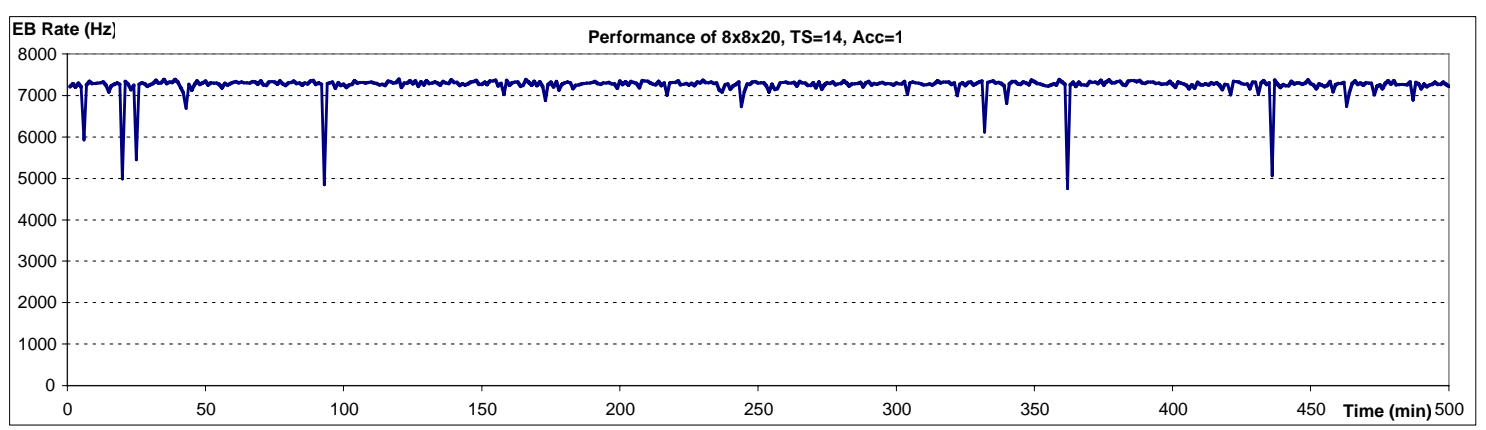

- Test with Event Filter. Real algorithms (Online 10.0.06) with ROS emulation sending Geant events

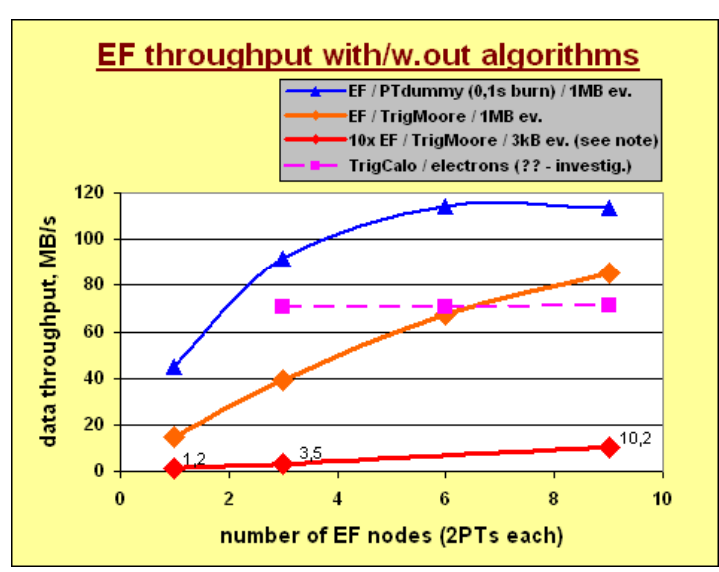




\section{DCS components}

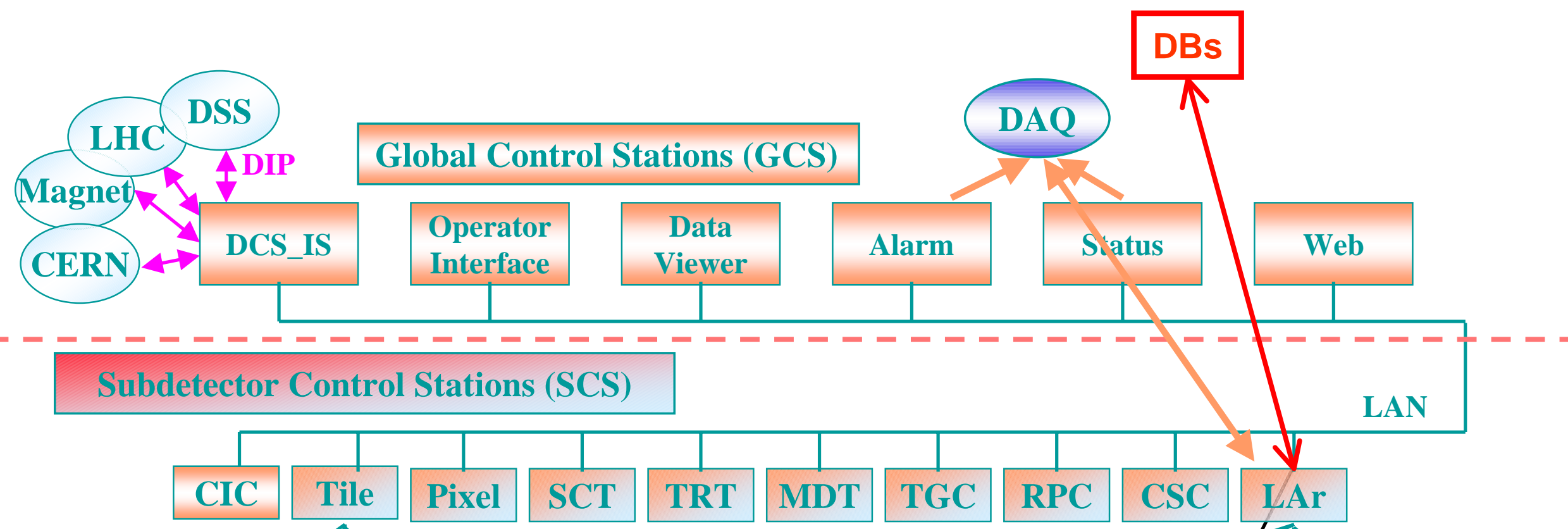

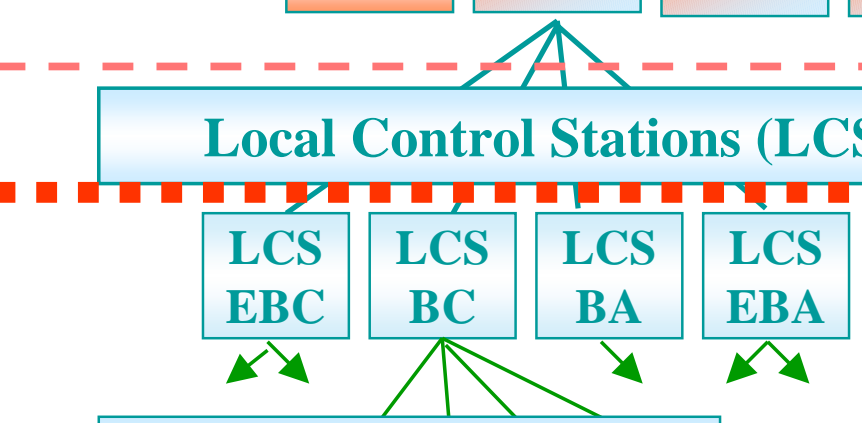

Front-End Systems

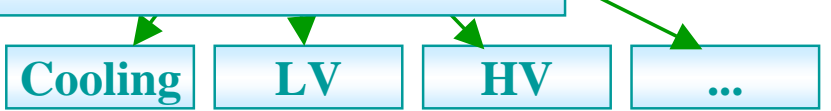

PVSS Connection X Pisa Meeting, 22 May 06

\section{$\longrightarrow \quad$ CAN Fieldbus}

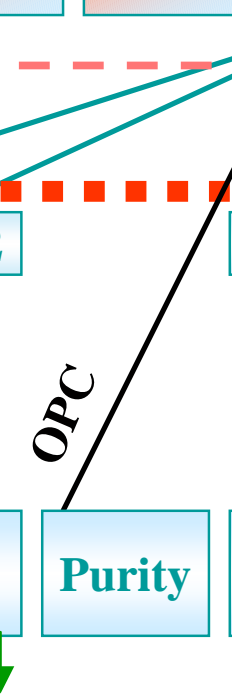




\section{Grouping of Sulb-System Partitions}

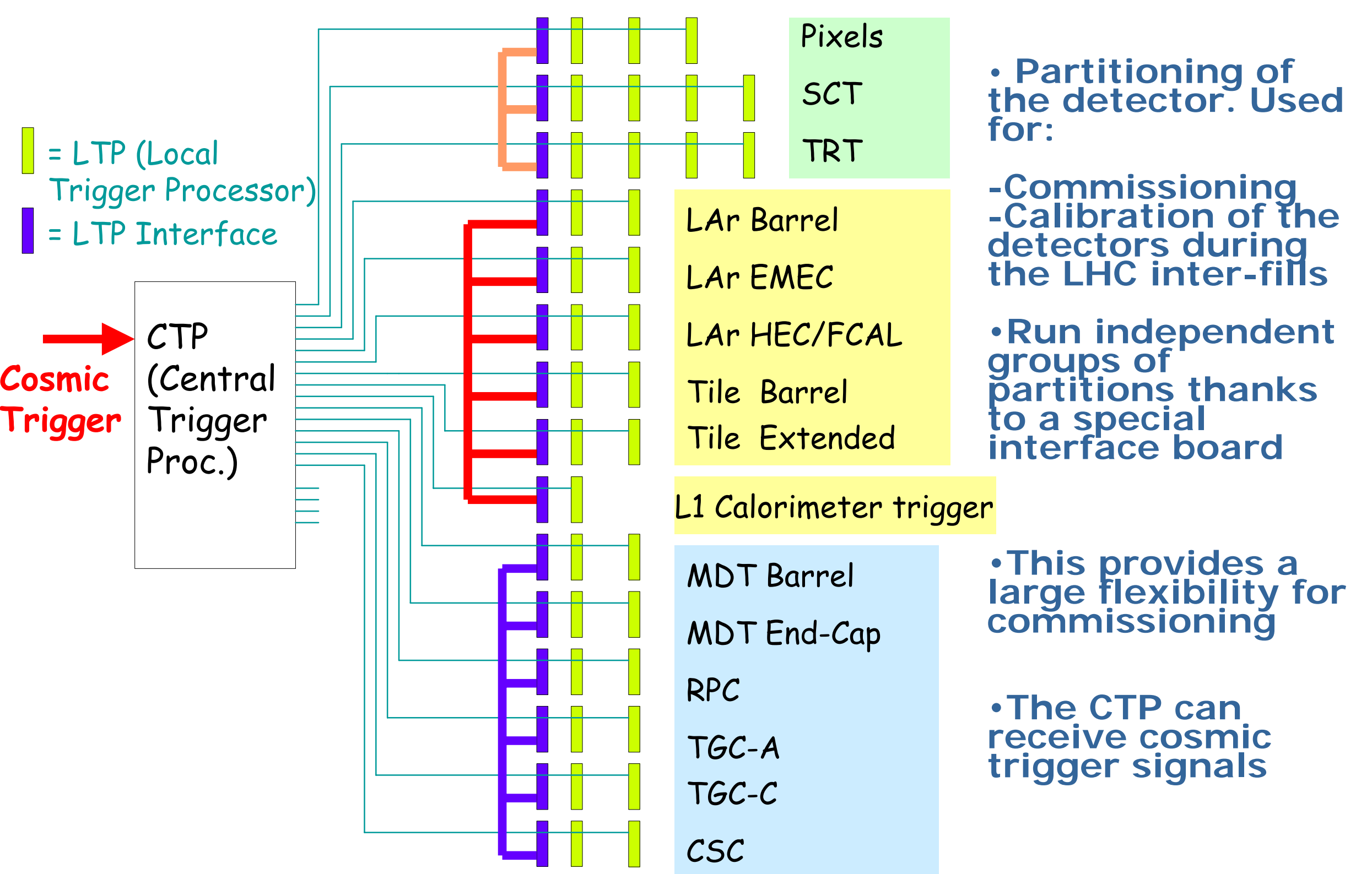


Calorimeter trigger

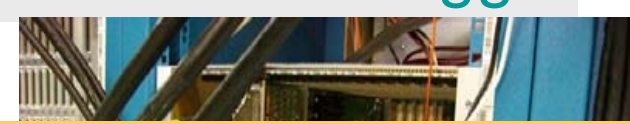
J une 06: final RODs

J uly 06: ROiBuilder, HLT

Sept 06: CTP integration VIII:
Muon Barrel trigger

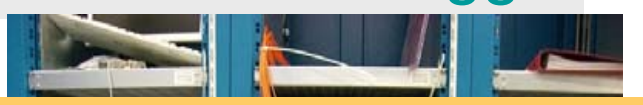

J une 06: run with lower sector, CTP, HLT cosmics

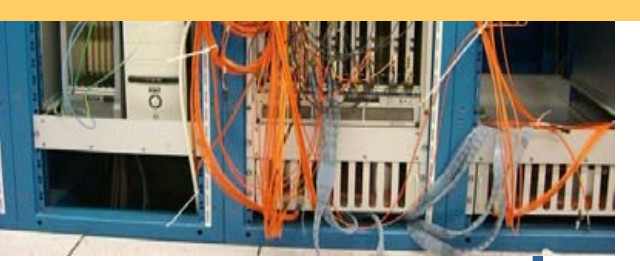

Muon Endcap trigger

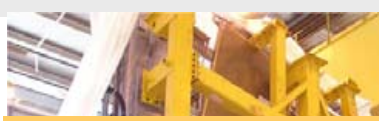

J uly 06: electronics for the TGC trigger (M1-C)

Sept 06: first final sector logic

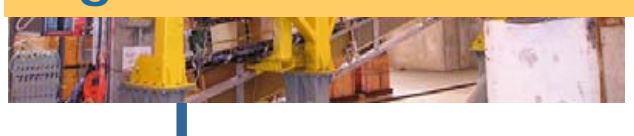

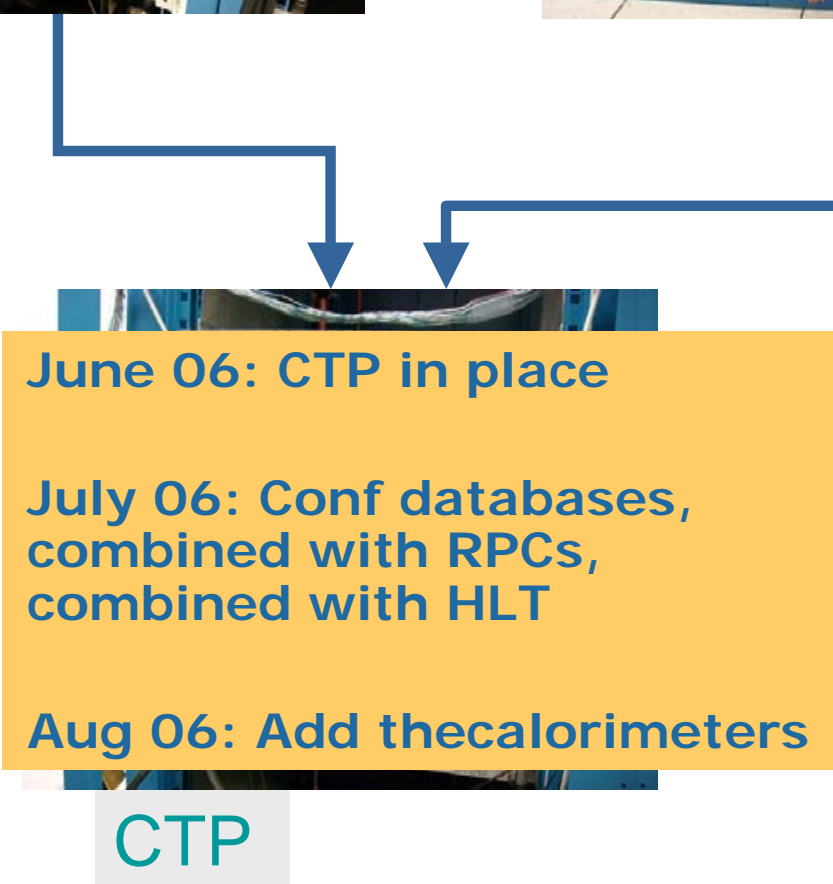

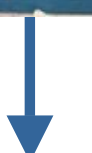




\section{Cosmic runs}

- Exercise the full functionalities:

- Conf DB, Trigger, DAQ, Slow control, HLT, on-line monitoring, event display, control room, shifts

- Full calibration procedures. Treatment of the bad channels

- Detectors available:

- LARG barrel, TILES barrel (limited readout). LICALO trigger

- Muon spectrometer lower sector

- Physics goals

- Amplitude inter calibration

- Timing studies

- Bad channels characterization

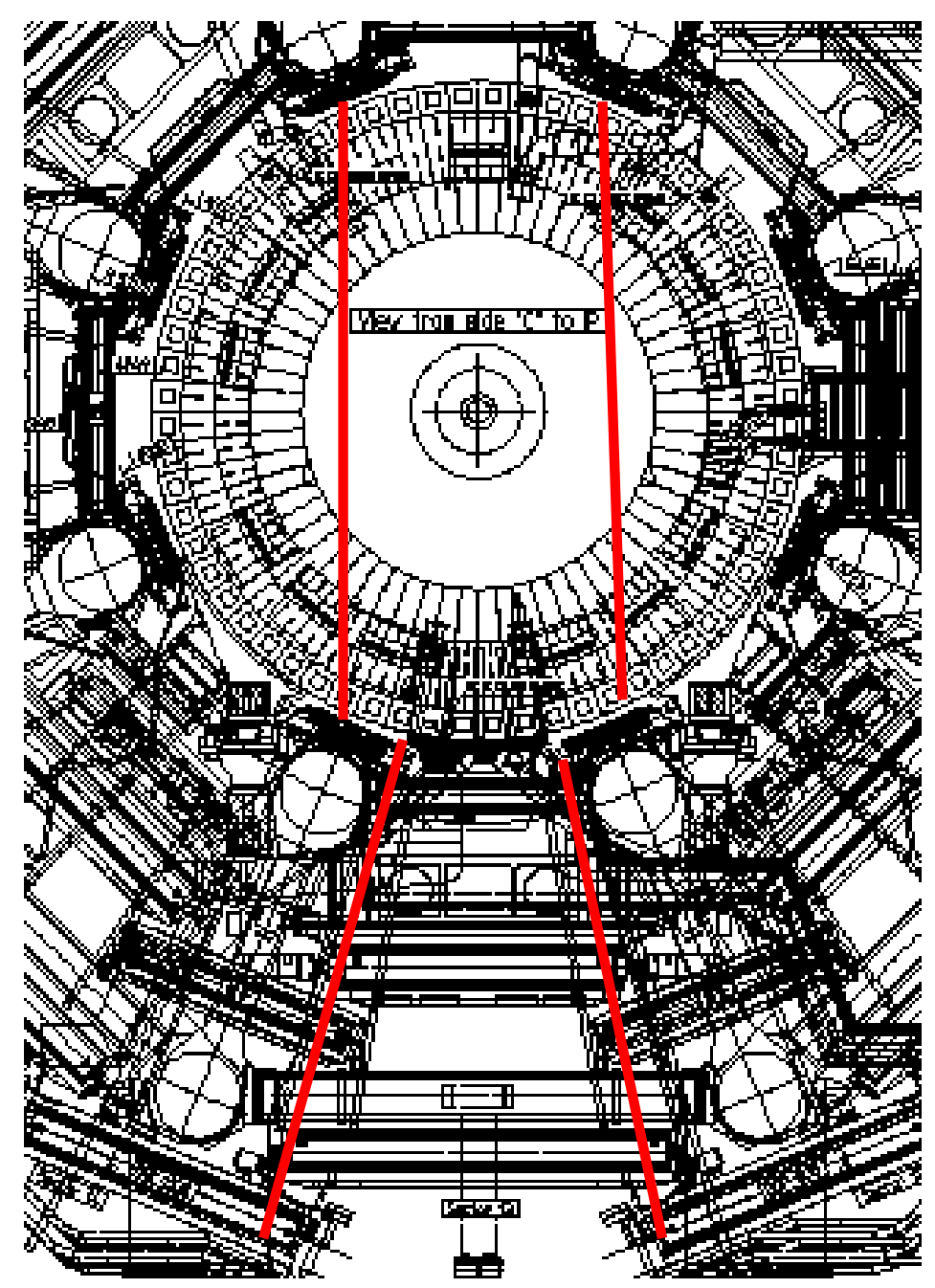




\section{Cosmic trigger with Tilles callo.}

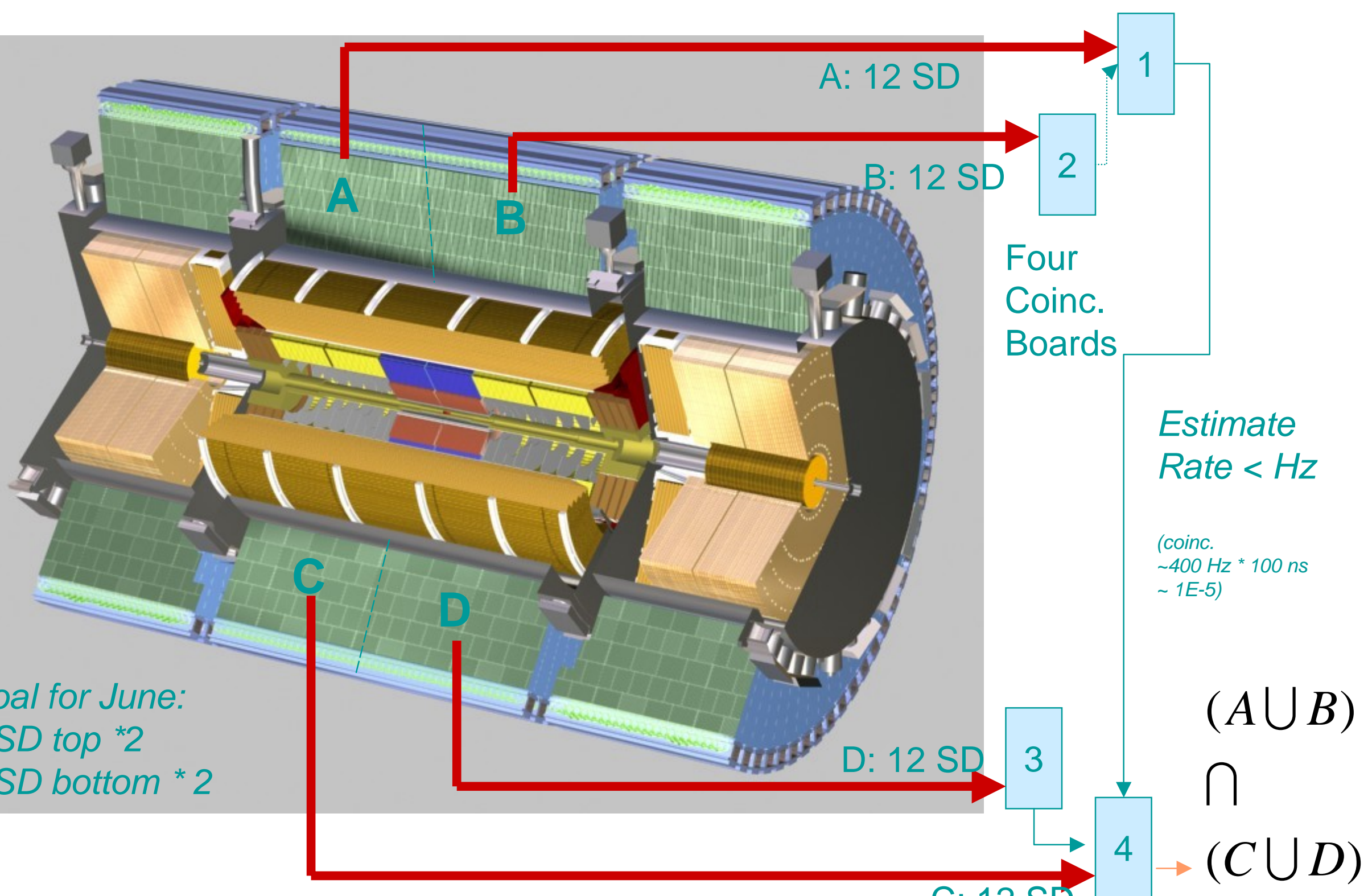




\section{Cosmic runs}

- Internal LARG inter-calibration

- Amplitude vs $\eta$

- Needs 40000 muons/cell for $0.5 \%$ precision

- Rate 0.04(0.15 non projective) Hz

- 100 muons/cell -> 100 days of DAQ

- But can understand timing at 0.6 ns

- TILES response to MIP

- At the combined test beam

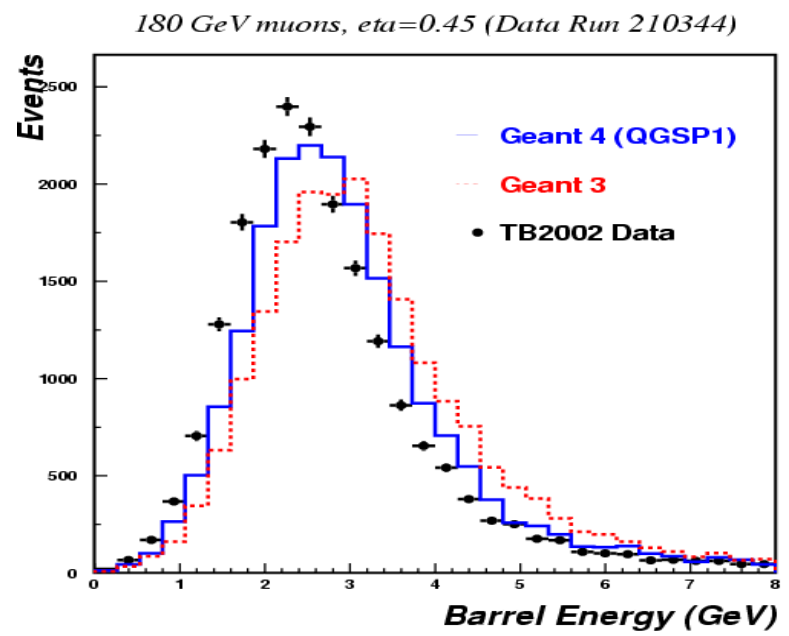

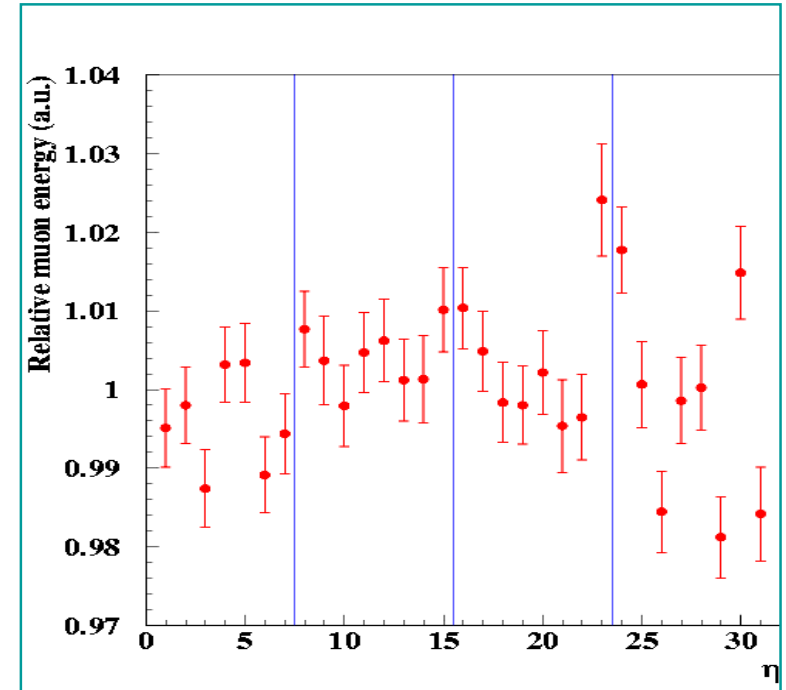

In the Cavern (LV power supplies different - >noise

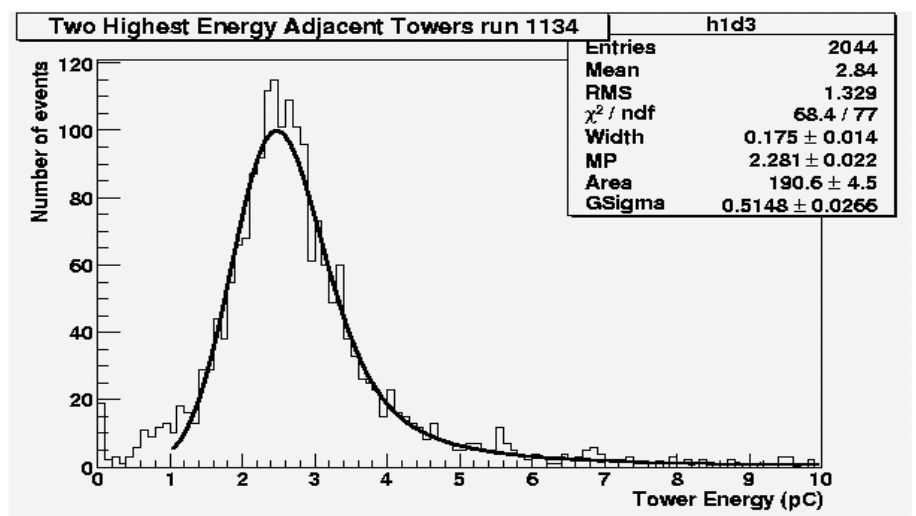


- LARG-TI LES inter-calibration

- Response to MIP at the combined test beam, compared to simulation, for layers and total

- $3 \%$ agreement
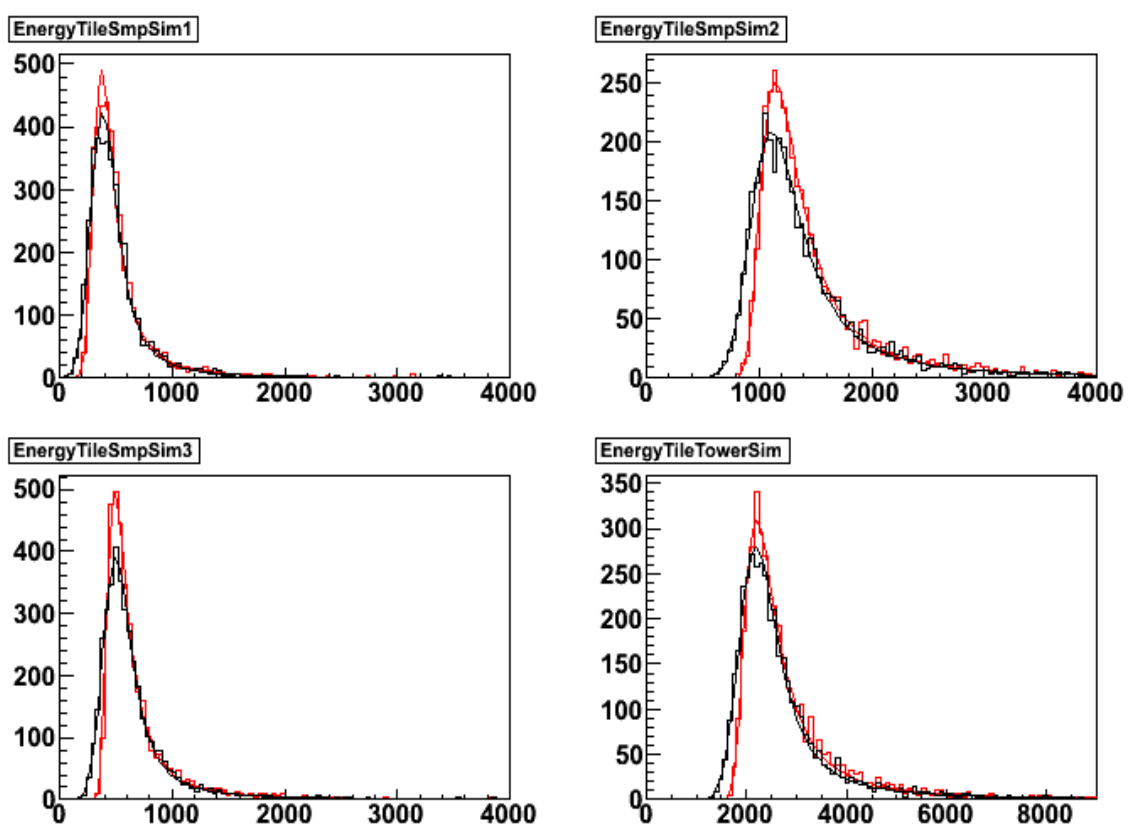

- TI LES timing

- From comparison of various cells

- Time resolution found of 1.7 ns

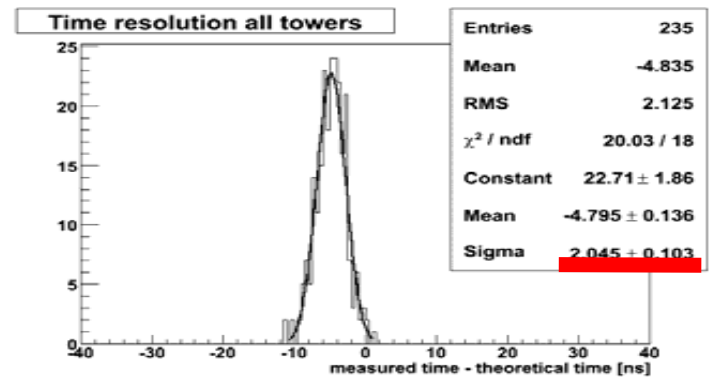

- TILES time difference (topbottom)

- After correction fits with the geometrical estimate

- Precision of $1.8 \mathrm{~ns}$

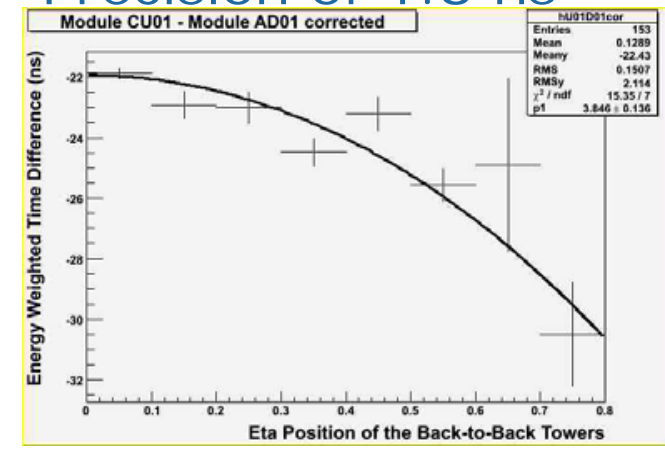

ATLAS commissioning, P. Perrodo 
- TILES

- $1 / 16$ of the barrel: $1 \mathrm{~GB} /$ day

- LARG

- Electronic calibration ramps (100,000 channels in the barrel): 5.2TB (transparent), 42GB (averaged locally in the LARG DAQ)

- Calibration signals recording: 650 GB

- Cosmics at $10 \mathrm{~Hz}$

- TILES: $1.4 \mathrm{MB} / \mathrm{s}$

- LARG 15MB/s. Maximum recording $20 \mathrm{MB} / \mathrm{s}$

- Muons (lower sector)

- $20 \mathrm{~Hz}$ and $2 \mathrm{kB}$ /event

- Autumn 06:

- end cap calorimeter. Need the Event Builder to take the data flow. 


\section{Barrel toroid test}

- Test the BT as a separate object

\begin{tabular}{|c|l|c|c|c|c|}
\hline run & \multicolumn{1}{|c|}{ Goal } & $\begin{array}{c}\text { Current } \\
{[\mathrm{kA}]}\end{array}$ & $\begin{array}{c}\text { ramp time } \\
{[\text { hours] }}\end{array}$ & $\begin{array}{c}\text { total } \\
\text { [hours] }\end{array}$ & $\begin{array}{c}\text { recovery } \\
\text { [days] }\end{array}$ \\
\hline 1 & test at low current & 5 & 0.3 & 3.9 & -- \\
\hline 2 & test at 1/4 of full energy & 10 & 0.7 & 2.9 & -- \\
\hline 3 & test at 1/2 of full energy & 15 & 1.0 & 3.6 & -- \\
\hline 4 & test at 3/4 of full energy & 18 & 1.3 & 3.5 & -- \\
\hline 5 & test at full energy & 20.5 & 1.4 & 3.8 & -- \\
\hline 6 & fast dump low current & 5 & 0.3 & 0.9 & $? ?$ \\
\hline 7 & fast dump (quench) & 15 & 1.0 & 1.3 & $? ?$ \\
\hline 8 & steady state test & 20.5 & 1.4 & 11.8 & -- \\
\hline
\end{tabular}

- Exercise everything already installed in presence of magnetic field

- Infrastructure (LV, gas, cooling) already installed around the detector

- Operate the Front-End electronics: LARG, TILES calorimeter, Barrel Muons (MDT, RPC) chambers

- Muon spectrometer: Alignment system, precise measurement of the field ( $\mathrm{B}$ at $1-2 \mathrm{mT}$ for $\mathrm{BI}$ at $410^{-3}$ ), effect of the surrounding structures

- Take cosmics with muon spec., TILES and LARG

- Similar issues for the solenoid mapping 


\section{Global cosmic run to first beams}

- Toward a global cosmic run (spring 2007)

- Integrate the detectors and systems as they come, when they grow in size, debug the full chain from shifts to data analysis.

- Cosmics can be used for the barrel part. Use of cosmics for the end-caps is under investigation.

- Beam gas

- can be used for for the end-caps: alignment, timing, intercalibration.

- Run at high L1 trigger rate with real events. DAQ challenge.

- Very first collisions: detector debugging and performances

- With 10-100 pb-1, 104 Z->ee, Z-> $\mu$, also tt->blv bjj, Trackers, Calorimeters, muon alignment, jet energy scale and b-tag

- A lot of work and fun is coming!

Thanks to: L. Chevalier, R. Nikolaidou, L. Pontecorvo, R. Teuscher, G. Unel, Th. Wengler 